\title{
Evolution of the ISM in starbursting dwarf galaxies
}

\author{
Fabrizio Brighenti ${ }^{1}$ and Annibale D'Ercole ${ }^{2}$ \\ ${ }^{1}$ Dipartimento di Astronomia, Università di Bologna, Italy \\ ${ }^{2}$ Osservatorio Astronomico di Bologna, Italy
}

\begin{abstract}
We present a 2D-hydrodynamical simulation of the gas flow generated by a starburst in a dwarf galaxy. We focus in particular on the ejection efficiency both of the ISM and of the metals produced during the burst. It turns out that while the galaxy is able to retain most of the ISM, a significant fraction of the metals is lost, supporting the differential wind scenario. A new burst may occur after $\sim 0.5-1 \mathrm{Gyr}$, when the central gas surface density approaches the critical value, during the post starburst gas replenishment.
\end{abstract}

We performed a 2D-hydrodynamical simulation of the ISM in a starburst dwarf galaxy. We assume that the energy production lasts $30 \mathrm{Myr}$, and we follow the gas evolution up to $500 \mathrm{Myr}$. We aimed at understanding the gas flow in general, and the circulation of the metal rich stellar ejecta in particular, in order to test the differential galactic winds scenario often used in chemical evolution studies (Tosi 1998).

We started with a rotating isothermal ISM in equilibrium in a potential well generated by an infinitesimally thin stellar disk and by a spherical quasiisothermal dark halo. Details can be found in D'Ercole \& Brighenti (1999). As the starburst wind starts, a typical superbubble develops. While the inner shock remains spherical because of the high sound speed of the hot rarefied shocked wind gas, the outer shock runs quicker along the polar direction, where the ISM density profile is steeper. Thus the superbubble assumes a hourglass shape (Fig. 1). As the polar expansion accelerates, the outer dense cold shell of swept up ISM fragments because of Rayleigh-Taylor instabilities. The shock in the polar region moves through a less and less dense gas, becoming unobservable. On the equatorial plane, instead, the inner edge of the cold shell starts to recede toward the center $\sim 20 \mathrm{Myr}$ after the starburst energy injection ceases. $150 \mathrm{Myr}$ after the starburst the central region of the galaxy is filled again by cold ISM. The replenishment, however, proceeds rather slowly. After $200 \mathrm{Myr}$ the gas mass inside the galactic region is $0.025 \%$ of the initial ISM mass, and increases linearly up to $20 \%$ at the end of the simulation, after 500 Myr. The gas surface-density increases in pace with the mass, and we obtain $\Sigma \simeq 6 \times 10^{20} \mathrm{~cm}^{-3}$ at $500 \mathrm{Myr}$. Since the gas is still accreting at this time, we can reasonably assume that, after a time of the order of $1 \mathrm{Gyr}, \Sigma$ reaches the critical value $\Sigma_{\mathrm{cr}} \simeq 5-10 \times 10^{20} \mathrm{~cm}^{-3}$ for efficient star formation (e.g., Gallagher \& Hunter 1984). This time-interval between bursts is broadly consistent with many studies of star formation history in BCD galaxies, indicating that stars are formed through a sequel of short bursts of star formation separated by long quiescent periods ( $c f$. Tosi 1999).

We define the ISM ejection efficiency $f_{\mathrm{ISM}} \equiv M_{\text {lost }} / M_{0}$, where $M_{0}$ is the ISM mass initially present and $M_{\text {lost }}$ the mass of gas whose velocity or sound 


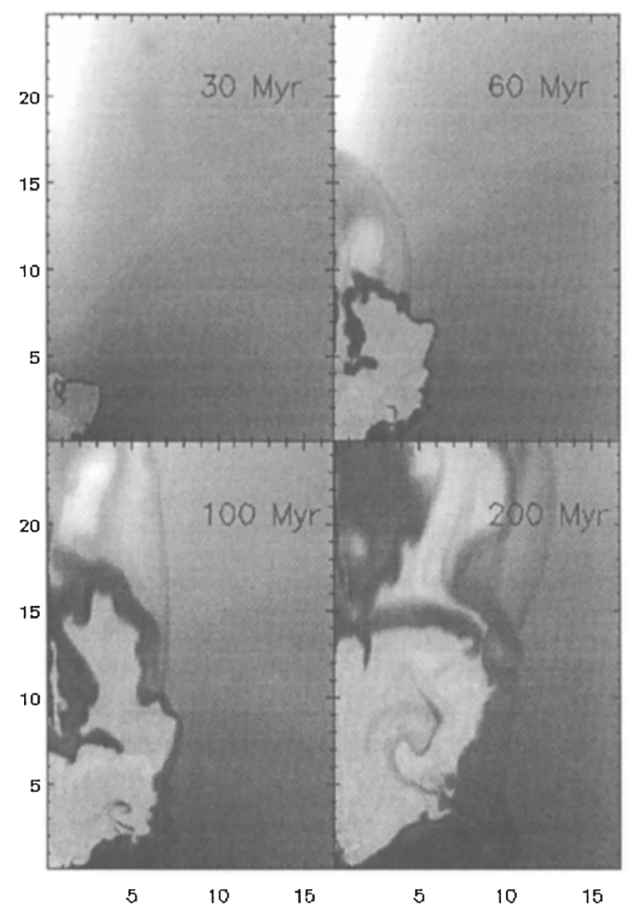

Figure 1. Map of the gas density distribution at four different times. Axis labels are in kpc. High density regions are darker.

speed is higher than the escape velocity. Analogously, the ejection efficency of the gas expelled by massive stars and SNII is $f_{\text {metals }} \equiv M_{\text {bur,lost }} / M_{\text {bur }}$, where $M_{\text {bur }}$ is the total gas mass returned by the burst. After 200 Myr we obtain $f_{\text {ISM }}=0.058$ and $f_{\text {metals }}=0.46$. Thus, the metal rich gas is lost by the galaxy much more easily than the pristine ISM (De Young \& Gallagher 1990). In fact, when a 'funnel' is formed through the galaxy, the wind leaves the galaxy through it without mixing efficiently with the ISM. Such dynamical behaviour supports the differential wind scenario required by most chemical models in order to explain the low $\alpha$-element abundances found in the irregulars.

\section{References}

D'Ercole, A., Brighenti, F., 1999, MNRAS in press

De Young, D.S., Gallagher, J.S. 1990, ApJ 356, L15

Gallagher, J.S., Hunter, D.A. 1984, ARAA 22, 37

Tosi, M. 1999, in: T.X. Thuan, C. Balkowski, V. Cayatte \& J. Tran Than Van (eds.) Dwarf Galaxies and Cosmology, Proc. XVIIIth Rencontre de Moriond (Editions Frontieres), in press (astro-ph/9806266) 